\title{
Modélisation du développement du phytoplancton dans une rivière canalisée eutrophe : le Lot (France)
}

\author{
A. Dauta1
}

Mots clés: Dynamique du phytoplancton, modèle numérique, rivière.

\begin{abstract}
Le modèle réalisé simule la dynamique d'une population phytoplanctonique composée de 8 espèces, sur le parcoúrs inférjeur ( $310 \mathrm{knn}$ ) d'une rivière eutrophe, en réponse aux conditions de température, lumière et nutriments. L'utilisation d'un modèle de type catastrophe appliqué aux conditions d'apparition d'un bloom de cyanophycées, de sa disparition et de ses effets sur les autres espèces est testé : celui-ci génère des phases de développement et de décroissance identiques à celles observées sur le milieu naturel considéré. De méme, l'évolution de la composition du phytoplancton (Diatomées. Cyanophycées, Chlorophycées) est reproduite. Ce modèle basé sur des mesures en laboratoire de constantes spécifiques de croissance des algues permet une approche des conditions de développement et de succession des algues.
\end{abstract}

Phytoplancton dynamics, numerical model, regulated river.

Keywords : Phytoplancton dynamics, numerical model, regulated river.

The model simulates the dynamics of a phytoplankton population with eight species in the lower course ( $310 \mathrm{~km}$ ) of an eutrophic river in relation to temperature, light and nutriments. A catastrophe model was tested with the conditions for the appearance of a bloom of Cyanophyceae, its decline and its effects on other species; the model generated phases of development and decline that were identical to those observed in the natural environment. Similarly, changes in the composition of the phytoplankton (diatoms, Cyanophyceae, Chlorophyceae) were reproduced. This model, based on laboratory estimates of specific growth rates of algae, provides a description of development conditions and succession for algae.

\section{Introduction}

L'aménagement des fleuves a pour effet d'augmenter simultanément la profondeur et le temps de rétention, ce qui permet l'établissement d'un phytoplancton. L'eutrophisation des eaux courantes est une variante de celle des lacs : l'écoulernent permanent de l'eau à travers le système donne à l'eutrophisation un caractère transitoire différé vers l'aval, en réponse à des fertilisations variables au long de la rivière.

Une rivière étudiée depuis 10 ans par le Labora. toire d'Hydrobiologie a servi de support à un essai de modélisation du développement du phytoplancton sur la partie inférieure de son cours. Le modèle réalisé se base sur des études écophysiologiques en laboratoire de la croissance des algues : il met en compétition 8 espèces, et utilise un sous-modèle de

1. Laboratoire d'hydrobiologie, UA CNRS 695, Université Paul Sabatier, 118, route de Narborne, 31062 Toulouse Cédex, France. type catastrophe pour simuler la formation de blooms de cyanophycées et leurs effets toxiques sur les autres espèces au moment de leur disparition.

Le modèle permet en particulier d'étudier l'influence du débit d'étiage sur l'évolution de la biomasse et la composition de la population phytoplanctonique.

\section{Caractéristiques du milieu modélisé : le Lot}

\subsection{Base de données}

La rivière Lot (fig. 1) a fait l'objet d'une étude approfondie au cours des dix dernières années : Dauta (1975), Dauta \& al. (1975), Massio (1976), Denat (1977), Angelier \& al. (1978), Capblancq et Dauta (1978), Tourenq \& al. (1978). Plusieurs essais de modélisation ont déjà été proposés sur des fractions de son cours: Capblancq \& Décamps (1978), Casanova-Batut (1977), Caussade \& al. (1978), Delclaux (1980), Dauta \& Capblancq (1985). En raison des bases de données existantes et de l'expérience 

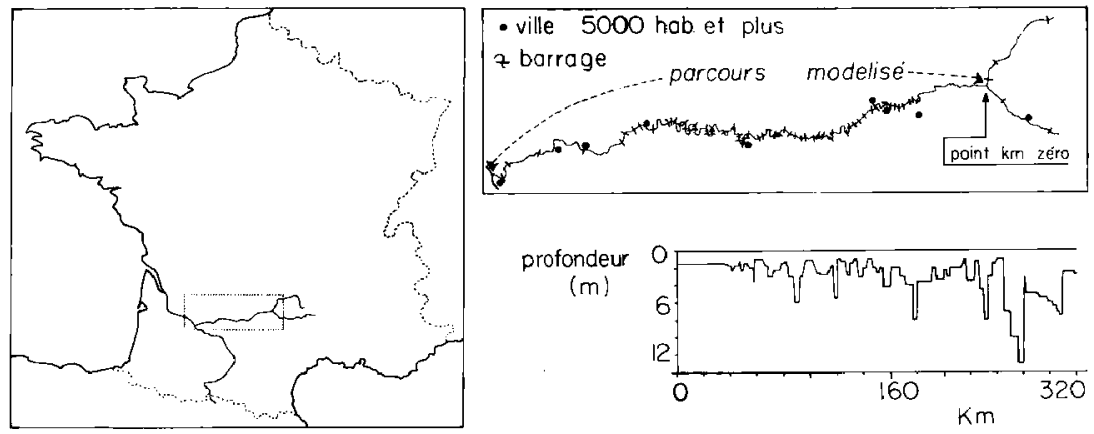

Fig. 1 : Présentation du milieu modelisé : le Lot.

acquise, un essai de modélisation sur la totalité de la nartie aval de la rivière $(310 \mathrm{~km})$ est réalisé ici.

\subsection{Morphométrie et hydrodynamique}

Après une portion de $50 \mathrm{~km}$ non canalisée, peu profonde et rapide, suivant le confluent du Lot avec son principal affluent la Truyère, le Lot se présente comme une succession de 62 biefs de caractéristiques très variables (fig. 1). Un modèle mathématique des écoulements (Sogreah 1975) permet de déterminer la vitesse moyenne par bief, pour un débit donné. Le tableau I donne les temps de séjours moyens pour 6 cas de débits régularisés.

\subsection{Traceurs biologiques}

La minéralisation des eaux augmente vers l'aval, avec des variations essentiellement déterminées par les fluctuations de débit de l'affluent principal et les apports des villes. Les teneurs minimales en nutriments ( $\mathrm{SiO} 2, \mathrm{NN} 03, \mathrm{PPO4}$ ), à la fin du printemps et en été, correspondent aux débits d'étiage et à la période de croissance du phytoplancton. Les apports diffus des régions agricoles sont tout aussi difficiles à évaluer que les rejets ponctuels des industries et des grandes villes.

Les biomasses d'algues observées dans la partie canalisće de la rivière donnent des profils longitudinaux irréguliers (fig. 2a), révélant des phases de croissance et de déclin, liées à l'historique des masses d'eau.

Le phytoplancton est principalement représenté par trois groupes: Diatomées, Cyanophycées et Chlorophycées. La composition moyenne de la population d'un profil longitudinal au cours de l'été est donnée par la figure $2 b$.

Il faut noter que sur la plus grande partie du cours de la rivière le zooplancton ne prend jamais une grande importance.

\section{Structure du modèle}

\section{I. Caractéristiques du modèle}

\subsubsection{Hypothèses générales}

Si l'on se réfère à la classification de Chahuneau et al. (1980), le modèle utilisé (diagramme fig. 3) est un modèle à niveaux trophiques subdivisés, dont la représentation s'inspire de ceux réalisés par Lehman \& al. (1975) ou Bierman (1976). Le phytoplancton est représenté par 8 espèces (cf. Tableau VII) : 6 chlorophycées, 1 diatornée et une cyanophycée fixatrice d'azote.

La quasi totalité des processus sont reliés à la tem. pérature soit par des fonctions complexes (VCmax, Ik, MUopt), linéaires (dégradation) ou affines (Vitesses maximales d'assimilation et $\mathrm{Ks}$ associés). La croissance et l'assimilation sont des phénomènes 


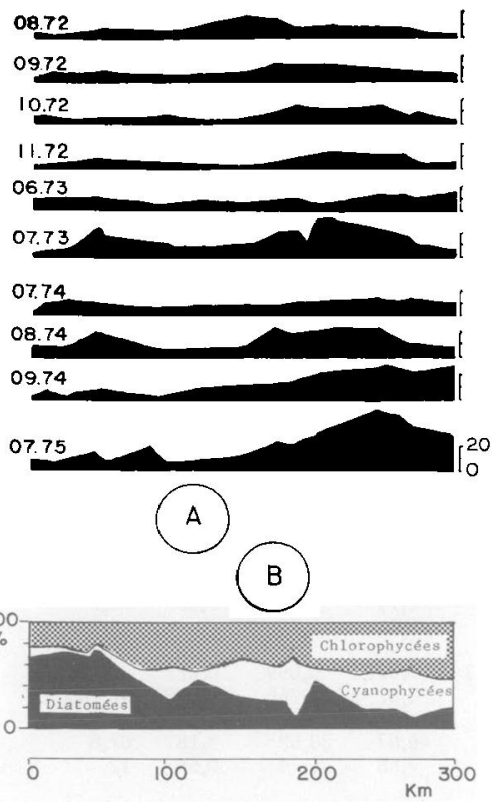

Fig. 2 : Valeurs observées : (a) Profils longitudinaux de chlorophylle $(\mu \mathrm{g} / 1)$. (b) Composition moyenne de la population.

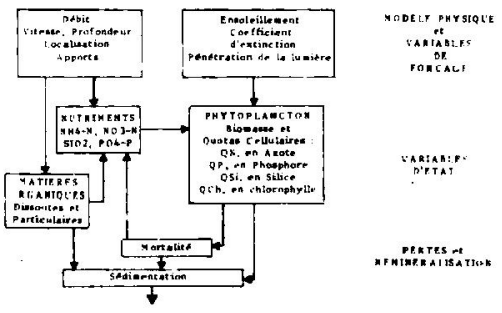

Fig. 3: Diagramme du modèle
Tableau I : Temps de séjour en fonction du débit sur le parcours considéré $(310 \mathrm{~km})$

\begin{tabular}{llllllr}
\hline Débit en $\mathrm{m}^{3 / \mathrm{s}}$ & 10 & 20 & 30 & 40 & 50 & 100 \\
Temps de séjour en & 87 & 46 & 32 & 25 & 18 & 11
\end{tabular}
jours

découplés. Le taux de croissance instantané est sous la dépendance des quotas en nutriments (Azote, Phosphore, Silice) selon une loi de réduction du minimum, et du quota en carbone. Défini par la stoechiométrie des cellules vis-à-vis des nutriments, l'état physiologique des cellules a une action sur la mortalité, la sédimentation et la teneur en chlorophylle (le bilan photosynthétique est calculé à chaque pas de temps en raison des variations de quota cellulaire en chlorophylle et de profondeur).

Du point de vue physique, la masse d'eau est supposée homogène : les apports sont réalisés sans loi de mélange et il n'est pas envisagé de stratification possible de la colonne d'eau, excepté dans le cas des Cya. nophycées dont le comportement de croissance est interprété à l'aide d'un sous-modèle de type catastrophe (cf $\S 3.3$ ).

\subsubsection{Variables d'étaı}

Pour 8 espèces et 3 nutriments, le modèle considère 15 variables d'état, dont 6 sont des variables de tableau indicées par rapport à l'élément de discrétisation (espèce considérée). Ces variables sont calculées par le système après initialisation au point $\mathrm{km}$ zéro:

Eléments nutritifs : SIO2, NNO3, NNH4, PPO4.

Fractions organiques d'origines exogènes et algues mortes.

Composition des cellules (réserves intracellulaires en nutriments, quota en carbone, teneur en chlorophylle).

Biomasse d'algues: nombre total de cellules, Chlorophylle.

\subsubsection{Variables de forçage}

Ces quantités variables sont définies par rapport au temps (Intensité lumineuse maximale journalière en surface) et à l'espace : apports en nutriments et rejets urbains ou industriels, hausse de température, vitesse de courant et profondeur des biefs traversés. 
Tableau II : Constantes utilisées pour le calcul des paramètres ou processus relatifs aux 8 espèces d'algues. Chlorophycées (esp. 1 à 6), Cyanophycée (esp. 7), Diatomée (esp. 8).

Unités: $\mathrm{Tj}=$ Température en ${ }^{\circ} \mathrm{C}, \mathrm{VC} \max =\mathrm{mg} \mathrm{C} / \mathrm{mg} \mathrm{chl.a/h}$. IK $\max ={ }_{\mu} \mathrm{E} / \mathrm{m}^{2} / \mathrm{s}, \mathrm{AVj}$ et $\mathrm{BVj}=$ vitesse en $\mu \mathrm{g}$-at de $\mathrm{j} / 10^{\circ} \mathrm{c} / \mathrm{h}, \mathrm{AKj}$ et $\mathrm{BKj}=$ concentration en $\mu \mathrm{g}$-at de $\mathrm{j} / \mathrm{litre}$, Quotas en $\mathrm{N}$ et $\mathrm{P}$ en $\mu \mathrm{g}-\mathrm{at} / 10^{\circ} \mathrm{c}$, Ouotas en Carbone en $\mathrm{mg} \mathrm{C} / 10^{9} \mathrm{c}, \mu \mathrm{m}=1 \mathrm{j}$

VSED $=/ \mathrm{m} / \mathrm{j}$

\begin{tabular}{|c|c|c|c|c|c|c|c|c|}
\hline Constantes & esp 1 & esp 2 & $\operatorname{esp} 3$ & $\operatorname{esp} 4$ & esp 5 & esp 6 & esp 7 & esp 8 \\
\hline $\begin{array}{l}\text { VCMAX } \\
\text { TinfVC } \\
\text { ToptVC } \\
\text { TsupVC }\end{array}$ & $\begin{array}{l}12 \\
10 \\
30 \\
40\end{array}$ & $\begin{array}{l}13 \\
12 \\
30 \\
40\end{array}$ & $\begin{array}{l}14 \\
13 \\
29 \\
40\end{array}$ & $\begin{array}{l}8,5 \\
14 \\
31 \\
40\end{array}$ & $\begin{array}{r}8 \\
12 \\
32 \\
40\end{array}$ & $\begin{array}{l}13 \\
16 \\
35 \\
40\end{array}$ & $\begin{array}{c}3,1 \\
7 \\
30 \\
40\end{array}$ & $\begin{array}{r}9 \\
5 \\
32 \\
40\end{array}$ \\
\hline $\begin{array}{l}\text { IK M AX } \\
\text { T infIK } \\
\text { T optIK } \\
\text { TsupIK }\end{array}$ & $\begin{array}{r}120 \\
0 \\
30 \\
40\end{array}$ & $\begin{array}{r}100 \\
4 \\
30 \\
40\end{array}$ & $\begin{array}{r}240 \\
6 \\
30 \\
40\end{array}$ & $\begin{array}{r}181 \\
4 \\
30 \\
40\end{array}$ & $\begin{array}{r}190 \\
8 \\
30 \\
40\end{array}$ & $\begin{array}{r}245 \\
12 \\
30 \\
40\end{array}$ & $\begin{array}{r}66 \\
0 \\
30 \\
40\end{array}$ & $\begin{array}{r}245 \\
5 \\
30 \\
40\end{array}$ \\
\hline $\begin{array}{l}\text { A VN } 03 \\
\text { B VNO3 }\end{array}$ & $\begin{array}{l}3,19 \\
-15,2\end{array}$ & $\begin{array}{c}7,35 \\
-85,5\end{array}$ & -16 & $\begin{array}{r}14,64 \\
-109,4\end{array}$ & $\begin{array}{l}11,14 \\
-71\end{array}$ & $\begin{array}{l}0,638 \\
8,8\end{array}$ & $\begin{array}{c}0,328 \\
-1,47\end{array}$ & $\begin{array}{r}7,5 \\
46,4\end{array}$ \\
\hline $\begin{array}{l}\text { AKN03 } \\
\text { BKNO3 }\end{array}$ & $\begin{array}{l}0,066 \\
1,22\end{array}$ & $\begin{array}{c}0,238 \\
-2,68\end{array}$ & $\begin{array}{l}0,102 \\
1,38\end{array}$ & $\begin{array}{r}0,20 \\
-2,25\end{array}$ & $\begin{array}{l}0,272 \\
-2\end{array}$ & $\begin{array}{c}0,108 \\
-0,17\end{array}$ & $\begin{array}{c}0,146 \\
-0,14\end{array}$ & $\begin{array}{l}0,165 \\
0,65\end{array}$ \\
\hline $\begin{array}{l}\text { A VPO4 } \\
\text { BVNO3 }\end{array}$ & $\begin{array}{c}0,97 \\
-5,7\end{array}$ & $\begin{array}{l}1,08 \\
6,8\end{array}$ & $\begin{array}{r}2,26 \\
-14,9\end{array}$ & $-10^{2,24}$ & $\begin{array}{l}1,74 \\
5,6\end{array}$ & $\begin{array}{l}0,39 \\
0,6\end{array}$ & $\begin{array}{l}0,36 \\
2\end{array}$ & $\begin{array}{l}0,6 \\
5,9\end{array}$ \\
\hline $\begin{array}{l}\text { AKP O4 } \\
\text { BKP 04 }\end{array}$ & $\begin{array}{r}0,054 \\
-0,395\end{array}$ & $\begin{array}{l}0,029 \\
0,34\end{array}$ & $\begin{array}{r}0,043 \\
-0,225\end{array}$ & $\begin{array}{c}0,043 \\
-0,17\end{array}$ & $\begin{array}{l}0,022 \\
0,02\end{array}$ & $\begin{array}{l}0,059 \\
0,265\end{array}$ & $\begin{array}{l}0,027 \\
0,178\end{array}$ & $\begin{array}{r}0,031 \\
-0,165\end{array}$ \\
\hline $\begin{array}{l}\text { QMP } \\
\text { QOP }\end{array}$ & $\begin{array}{l}8,33 \\
1,24\end{array}$ & $\begin{array}{c}11,63 \\
1,9\end{array}$ & $\begin{array}{l}6,06 \\
0,87\end{array}$ & $\begin{array}{r}41,41 \\
7,33\end{array}$ & $\begin{array}{r}46,87 \\
7,56\end{array}$ & $\begin{array}{r}30,62 \\
1,73\end{array}$ & $\begin{array}{l}3,18 \\
0,54\end{array}$ & $\begin{array}{l}67,8 \\
12\end{array}$ \\
\hline $\begin{array}{l}\text { QMN } \\
Q O N\end{array}$ & $\begin{array}{l}393 \\
120\end{array}$ & $\begin{array}{r}196 \\
81\end{array}$ & $\begin{array}{r}126 \\
84\end{array}$ & $\begin{array}{l}378 \\
170\end{array}$ & $\begin{array}{l}260 \\
180\end{array}$ & $\begin{array}{l}93 \\
40\end{array}$ & $\begin{array}{r}132 \\
57\end{array}$ & $\begin{array}{l}531 \\
161\end{array}$ \\
\hline $\begin{array}{l}Q M C \\
Q O C\end{array}$ & $\begin{array}{l}34 \\
11,2\end{array}$ & $\begin{array}{l}100 \\
10,2\end{array}$ & $\begin{array}{l}85 \\
17\end{array}$ & $\begin{array}{l}72 \\
24\end{array}$ & $\begin{array}{r}144 \\
12\end{array}$ & $\begin{array}{r}20 \\
2\end{array}$ & $3^{7,5}$ & $\begin{array}{l}84 \\
34\end{array}$ \\
\hline $\begin{array}{l}Q C \text { himax } \\
Q C h 10 N \\
Q C h 10 P \\
K Q N \\
K Q P\end{array}$ & $\begin{array}{c}430 \\
215 \\
290 \\
153 \\
13,4\end{array}$ & $\begin{array}{l}300 \\
150 \\
200 \\
34 \\
17,56\end{array}$ & $\begin{array}{l}500 \\
250 \\
340 \\
-42 \\
10,15\end{array}$ & $\begin{array}{l}950 \\
475 \\
640 \\
38 \\
63,03\end{array}$ & $\begin{array}{r}1120 \\
560 \\
750 \\
-100 \\
72,12\end{array}$ & $\begin{array}{c}114 \\
57 \\
80 \\
13 \\
66,24\end{array}$ & $\begin{array}{l}120 \\
100 \\
80 \\
318 \\
4,74\end{array}$ & $\begin{array}{l}900 \\
450 \\
600 \\
209 \\
9,6\end{array}$ \\
\hline $\begin{array}{l}\mu m \\
\text { T inf } \\
\text { T opt } \\
\text { T sup }\end{array}$ & $\begin{array}{l}1,8 \\
15 \\
31 \\
45\end{array}$ & $\begin{array}{l}1,75 \\
11 \\
32 \\
40\end{array}$ & $\begin{array}{l}1,85 \\
14 \\
30 \\
40\end{array}$ & $\begin{array}{l}1,8 \\
15 \\
31 \\
40\end{array}$ & $\begin{array}{l}1,3 \\
14 \\
32 \\
42\end{array}$ & $\begin{array}{l}1,95 \\
14 \\
34 \\
45\end{array}$ & $\begin{array}{l}0,75 \\
10 \\
33 \\
45\end{array}$ & $\begin{array}{l}1,22 \\
5 \\
22 \\
32\end{array}$ \\
\hline $\begin{array}{l}\text { VSEDmini } \\
\text { VSEDmaxi }\end{array}$ & $\begin{array}{l}0,05 \\
0,5\end{array}$ & $\begin{array}{l}0,05 \\
0,5\end{array}$ & $\begin{array}{l}0,05 \\
0,25\end{array}$ & $\begin{array}{l}0,05 \\
0,5\end{array}$ & $\begin{array}{l}0,05 \\
0,5\end{array}$ & $\begin{array}{l}0,05 \\
0,25\end{array}$ & $\begin{array}{l}0,05 \\
0,25\end{array}$ & $\begin{array}{l}0,05 \\
0,5\end{array}$ \\
\hline $\begin{array}{l}\text { A VSI02 } \\
\text { B VSIOO2 }\end{array}$ & & & & & & & & $\begin{array}{l}15,2 \\
-44\end{array}$ \\
\hline $\begin{array}{l}\text { AKSIO2 } \\
\text { BKSIO2 }\end{array}$ & & & & & & & & $\begin{array}{r}0,27 \\
-1,35\end{array}$ \\
\hline $\begin{array}{l}\text { QMSI02 } \\
\text { Q0SIO2 }\end{array}$ & & & & & & & & $\begin{array}{l}657 \\
300\end{array}$ \\
\hline
\end{tabular}


Tableau III : Valeurs des constantes communes à tous les processus.

\begin{tabular}{|c|c|c|c|}
\hline Constante & Valeur & Unité & Définition \\
\hline A & 0,015 & $/ \mathrm{m}$ & Coefficient d'extinction des algues \\
\hline B & 1,3 & / $\mathrm{m}$ & Coefficient d'extinction de l'eau \\
\hline $\mathbf{R}$ & 0,10 & & Quotient de respiration. \\
\hline MORTAmax & 0,1 & $/ \mathbf{j}$ & Taux de mortalité maximal \\
\hline$M \cup \min$ & 0,1 & & Contrôle du taux de mortalité \\
\hline VSEDpart & 0,5 & $/ \mathrm{m} / \mathrm{j}$ & $\begin{array}{l}\text { Taux de sédimentation des matières } \\
\text { organiques \# algues }\end{array}$ \\
\hline CHLABLOOM & 80 & $\mu \mathrm{g} / \mathrm{l}$ & $\begin{array}{l}\text { Concentration seuil de chlorophylle doe aux } \\
\text { cyanophycées, pour laquelle il y a bloom. }\end{array}$ \\
\hline JANABIOSE & 1 & $\mathbf{j}$ & $\begin{array}{l}\text { Délai après lequel la mort du bloom de } \\
\text { cyanophycée exerce un effet toxique. }\end{array}$ \\
\hline JBLOOMTOX & 4 & j & Nombre de jours de toxicité du bloom. \\
\hline $\mathrm{K} 1 \mathrm{~N}$ & 0,005 & ${ }^{\circ} \mathrm{C} / \mathrm{j}$ & Dégradation des matières organiques $(->N N H 4)$ \\
\hline $\mathrm{K} 2 \mathrm{~N}$ & 0,008 & ${ }^{\circ} \mathrm{C} / \mathrm{j}$ & Taux de Nitrification (NNH4 $\rightarrow$ NN03) \\
\hline $\mathrm{K} 3 \mathrm{~N}$ & 0,001 & ${ }^{\circ} \mathrm{C} / \mathrm{j}$ & Taux de Dénitrification \\
\hline$k 1 P$ & 0,005 & ${ }^{\circ} \mathrm{C} / \mathrm{j}$ & Dégradation des matières organiques (-> PP 04) \\
\hline
\end{tabular}

L'intensité maximale en surface $\left(\mathrm{IO}_{2}\right.$ au temps t est calculée d'après le système d'équations suivant : $\mathrm{IO}_{\mathrm{h}}=\operatorname{Imax}_{\mathrm{j}}^{*} \sin \left(3.14^{*}\right.$ (h-HL) PHOTOPERIODE))

(PHOTOPERIODE $=12.4^{*} \operatorname{SIN}\left(3.14^{*}(\mathrm{~J}+75) / 360\right)$ $\mathrm{HL}=12$ - PHOTOPERIODE $/ 2$

où h = heure à l'instant $t$ et $\operatorname{Imax}_{\mathrm{j}}$ l'intensité maximale de surface pour le jour $\mathrm{J}$.

\subsubsection{Paramètres}

Ce modèle comporte plus de 200 paramètres, dont les principaux sont :

* pour les algues :

+ les vitesses d'assimilation des éléments nutri. tifs et les constantes de demi-saturation associées.

+ les valeurs maximales et minimales des quotas cellulaires en nutriments, en carbone, et en chlorophylle.
+ le coefficient d'extinction de la lumière, le taux maximal de photosynthèse et la valeur Ik associée.

+ le taux de croissance optimal,

+ et pour les pertes : le taux de mortalité, de respiration et de sédimentation.

* pour les fractions non algales : la biodégradation et lex pertes par sédimentation.

Les tabivaux II et III donnent les valeurs des constantes et des paramètres pour les divers processus.

\subsection{Analyse du système}

\subsubsection{Formulation des processus (Tableau IV)}

Le taux de croissance optimal (MUopt) est défini comme une fonction de la température en utilisant la formulation de Lehman et al. (1975):

MUopt $=\mu \mathrm{m}^{*} \exp \left(-2.3^{*}(\mathrm{~T}-\mathrm{Top} t)^{2} /(\mathrm{Ts}-\mathrm{Top} \mathrm{t})^{2}\right) \mathrm{si}$ T>Topt 
Tableau IV: Formulation des processus.

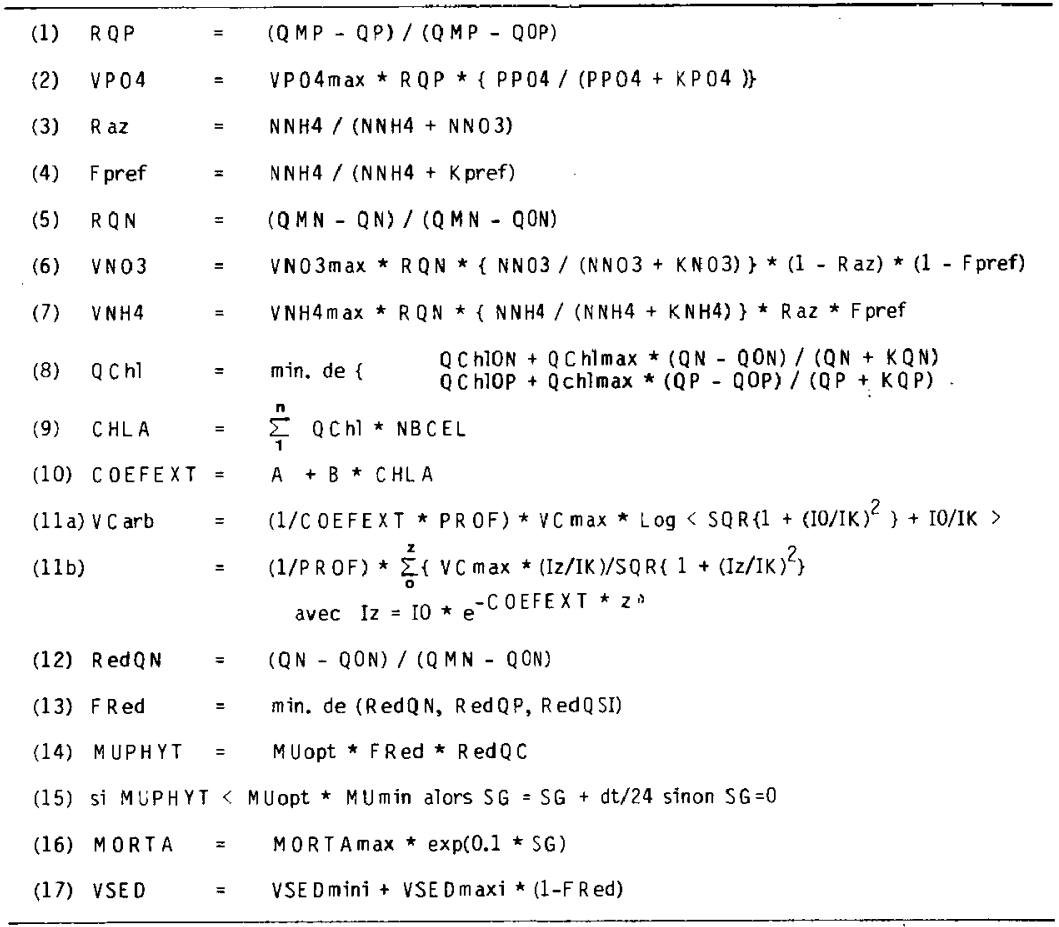

\section{T>Topt}

$=\mu \mathrm{m}^{\star} \exp \left(-2.3^{\star}(\mathrm{T}-\mathrm{Topt})^{2} /(\mathrm{Ti}-\mathrm{Top} t)^{2}\right) \mathrm{si}$

Où $\mu \mathrm{m}=$ taux maximal de croissance, Topt $=$ température correspondant à $\mu \mathrm{m}$,

Ts et $\mathrm{Ti}$, respectivement les températures supérieures et inférieures à Topt pour lesquelles $\mu={ }_{\mu} \mathrm{m}$ 10 ,

(cette formulation est également employée pour Ik et VCmax),

Des fonctions affines permettent d'exprimer les vitesses d'assimilation et les $\mathrm{Ks}$ associés, ex.
VNO3max $=$ AVNO3 ${ }^{*}$ TEMP + BVNO3

$\mathrm{KNO} 3=\mathrm{AKNO} 3{ }^{*}$ TEMP + BKNO3

L'assimilation des nutriments est décrite par une loi de Michaelis-Menten contrôlée en retour par l'état nutritionnel des cellules (ex. RQP pour le Phosphore).

L'existence d'une consommation préférentielle (Fpref) de NNH4 est prise en compte (équations 3 à 7).

Les profils de photosynthèse sont calculés avec un pas de temps maximal de 1 heure. En fonction du 
Tableau V: Expression des laux de variation des compartiments.

\begin{tabular}{|c|c|c|}
\hline (18) & $\mathrm{dNBCEL/dt}$ & $=$ MUPHYT - MORTA - VSED/PROF \\
\hline$(19 a)$ & $\mathrm{dQN} / \mathrm{dt}$ & $=($ VNO3 + VNH4) - MUPHYT * QN \\
\hline (19b) & $\mathrm{dQP} / \mathrm{dt}$ & $=$ VPO4-MUPHYT $\star Q P$ \\
\hline (19c) & $\mathrm{dQC/dt}$ & $=Q C H *\{V C a r b-Q C *(M U P H Y T-R * V C \max )\}$ \\
\hline$(20)$ & dNORGPHYT/dt & $=N B C E L \star Q N * M O R T A-N O R G P H Y T *(5 * K 1 N \star T E M P+V S E D P A R T / P R O F)$ \\
\hline (21) & dPORGPHYT/dt & $=N B C E L \star Q N \star M O R T A-P O R G P H Y T \star(5 \star K 1 P \star T E M P+V S E O P A R T / P R O F)$ \\
\hline$(22)$ & $\mathrm{dNORG/dt}$ & $=-K 1 N * T E M P-V S E D P A R T / P R O F$ \\
\hline (23) & $\mathrm{dPORG/dt}$ & $=-K 1 P * T E M P-V S E D P A R T / P R O F$ \\
\hline (24) & $\mathrm{dNN} 03 / \mathrm{dt}$ & $=N N H 4 * K 2 N * T E M P-V N 03 * N B C E L-K 4 * N N 03 / P R O F$ \\
\hline & $\times 4$ & $=K 3 N * \exp (0.158+0.065 * T E M P)$ \\
\hline$(25)$ & $\mathrm{dNNH4/dt}$ & 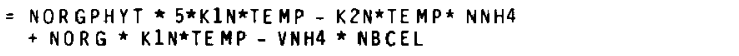 \\
\hline (26) & $d P P 04 / d t$ & $=P 0 R G P H Y T * 5 * K 1 P * T E M P+P O R G * K 1 P * T E M P-V P 04 * N B C E L$ \\
\hline
\end{tabular}

coefficient d'extinction de la lumière dans l'eau (COEFEXT) et la profondeur (PROF) à l'instant $t$, le modèle calcule le bilan photosynthétique de la colonne d'eau soit :

- par utilisation de la solution numérique de l'inté. gration de la photosynthèse de Talling (1957) (eq. 11a)

par cumulation de valeurs de photosynthèse par tranches d'eau (eq. 11b).

Le gain de biomasse est donné par le taux de crois. sance instantané MUPHYT (eq. 14), exprimé comme le taux optimal de croissance pour la température du iour considéré, et affecté de facteurs de réduction dus à une éventuelle carence en nutriments (loi du minimum, ex. : RedQN, eq. 12), et au quota en carbone.

Les taux de perte sont étroitement liés à l'état physiologique des cellules:

* la mortalité (MORTA) prend en compte (Lehman $\&$ al., 1975) le nombre de jours (SG) passés en conditions défavorables (MUopt * MUmin)

* la sédimentation (VSED) s'accélère lorsque les réserves s'épuisent (FRed).

\subsubsection{Expression des taux de variations}

Les taux de variation des différentes variables d'état Nombre de cellules, quotas cellulaires, nutriments. et compartiments particulaires) sont exprimés tableau V.

Les taux de biodégradation (K1N, K1P) de la matière organique issue des algues mortes (NORGPHYT, PORGPHYT) sont majorés (eq. 20 \& 21) par rapport à ceux des substrats organiques exogènes (NORG, PORG). A l'exemple de la Tamise (Thomson 1979), un terme prend en compte le processus de dénitrification (eq. 24) proportionnel à la surface de contact sédimenteau.

3.3. Variantes du sous-modèle traitant le cas de la Cyanophycée (Tableau VI)

L'assimilation de l'azote atmosphérique est décrite de façon implicite: lorsque la concentration (NH4-N + NO3-N) devient inférieure à $0,5 \mu$ g-at/litre, il est considéré que l'algue fixe N2 : son quota en azote tend alors vers le quota maximal. Les observations réalisées par Ogawa \& Car (1969), Fogg (1971), Horne \& al. (1979) ont servi de base à cette extrapolation.

Une deuxième particularité réside dans la modélisation de la vitesse de sédimentation. On a souvent cherché à relier la dominance des Cyanophycées en surface à leur flottabilité (Knoechel \& Kalff 1975). L'importance du dioxyde de carbone, pressentie par Kuentzel (1969), a été mise en évidence par Booker \& 
Tableau VI : Equations spécifiques au sous-modèle traitant du bloom de Cyanophycées et de ses conséquences.

\begin{tabular}{|c|c|c|}
\hline (27) & CYANOCONC & $=N B C E L \star Q C H L * P R O F / 0.3$ \\
\hline (28) & si CYANOCOnC? & $\begin{array}{c}\text { CHLABL OOM alors les conditions de bloom sont réalisées, } \\
\text { et les équations } 29 \text { à } 31 \text { sont appliquées. }\end{array}$ \\
\hline (29) & MORTA(cyano) $=$ & $=0.2$ \\
\hline (30) & JBLOOM & $\begin{aligned}= & J B L O O M+d t / 24,\{B L O O M O U R=\text { JANABIOSE + JBLOOMTOX }\} \\
& \text { si JBLOOM >BLOOMDUR alors fin des effets du sous-modèle }\end{aligned}$ \\
\hline (31) & $B L O 0 M E F F$ & $\begin{aligned}= & \sin \{3.14 *(J B L O O M-J A N A B I O S E) / J B L O O M T O X\} \\
& \text { si BLOOMEFF<0 alors BLOOMEFF }=0\end{aligned}$ \\
\hline (32) & MORTA(\#cyano) $=$ & $=$ MORTAmax * $\exp (0.1 * S G)+2 *$ MORTAmax *BLOOMEFF \\
\hline
\end{tabular}

Walsby (1981) et Klemer \& al. (1982), avec un lien évident quant à l'intensité lumineuse (Konopka \& al. 1978, Walsby \& Booker 1980). Ces diverses modalités du comportement des Cyanophycées ont été modélisées ainsi : si la valeur du quota cellulaire en carbone s'abaisse au dessous d'un seuil (fixé arbitrairement à 1.25 du quota minimal), alors la vitesse de chute est considérée comme nulle (ce test ayant priorité sur les carences éventuelles en azote ou phosphore). Ces deux contraintes de la modélisation tendent à favoriser la croissance d'une cyanophy. cée fixatrice d'azote.

En se basant sur les études réalisées par Duckstein \& al. (1979), il est possible d'écrire un modèle donnant un scénario de type catastrophe : à partir d'une concentration seuil (CHLABLOOM) fixée et atteinte par les cyanophycées concentrées dans la couche supérieure de la masse d'eau, on considère que le bloom de cyanophycées meurt (taux de crois. sance et processus sont nuls, la mortalité est constante $=0.2$ / jour). Dans le même temps, un effet toxique est appliqué aux autres espèces et s'ajoute à la mortalité naturelle. La phase de disparition du bloom dont les jours sont comptabilisés (JBLOOM), prend fin lorsque celui-ci atteint la valeur totale de iours fixee (BLOOMDUR). A cet instant, tous les effets dus à la mort du bloom sont annulés, et des cyanophycées peuvent éventuellement reprendre leur croissance.

\subsection{Conditions $d$ 'initialisation et de forçage}

La population phytoplanctonique initiale (point $\mathrm{Km}$ zéro) représente une biomasse de $5 \mu \mathrm{g}$ / litre de chlorophylle, dont la composition est identique à celle observée pendant la période estivale (Dauta 1975) : une loi de distribution du type Motomura où la diatomée occupe le rang 1 a été appliquée pour déterminer l'abondance relative des 8 espèces présentes.

Les teneurs en nutriments correspondent aux valeurs moyennes enregistrées sur ce point de la rivière; ces valeurs initiales étant relativement élevées, les divers quotas cellulaires des algues sont fixés aux valeurs maximales.

Le débit est supposé constant pendant toute la durée du déplacement de l'eau : à chaque pas de temps, le point $\mathrm{km}$ calculé définit ainsi la profondeur, la vitesse de déplacement et les apports éventuels par unité de volume.

\section{4. - Résultats}

Compte-tenu des conditions de modélisation qui évoquent des situations fictives (débit régularisé sur tout le temps de parcours) et qui s'appuient sur des moyennes saisonnières, et en l'absence de données de terrains correspondantes, il s'agit surtout de vérifier que les phénomènes simulés restituent globalement les événements enregistrés sur le terrain. 

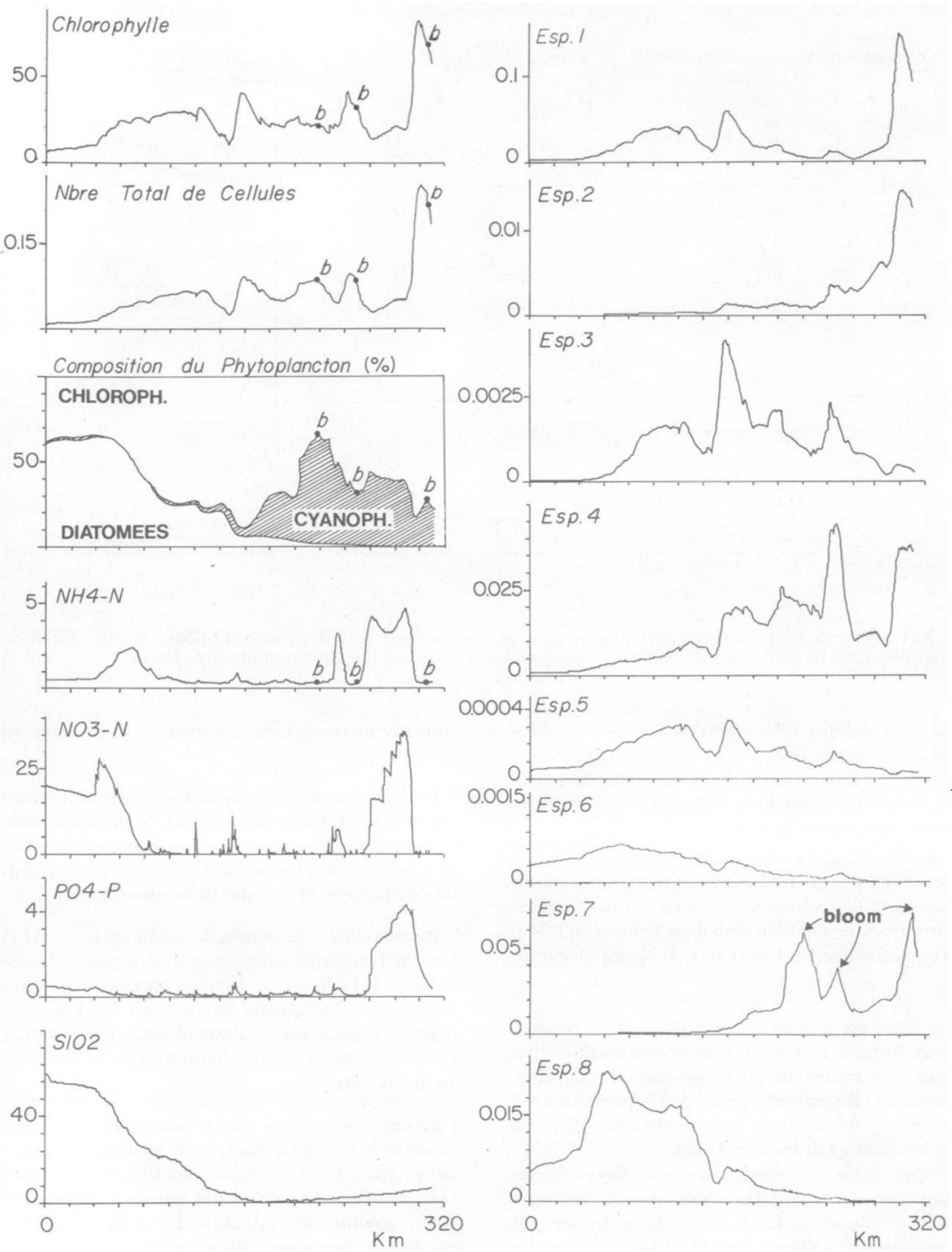

Fig. 4 : Simulation pour un débit de $10 \mathrm{~m}^{3 /}$ s. Date de départ $=$ ler juin. Ensemble des variables : Chlorophylle $(\mu \mathrm{g} / 1) \mathrm{Nutri}$. ments $\left(\mu \mathrm{g}\right.$-at/1), Nombre de cellules $\left(10^{9 / 1}\right)$. 

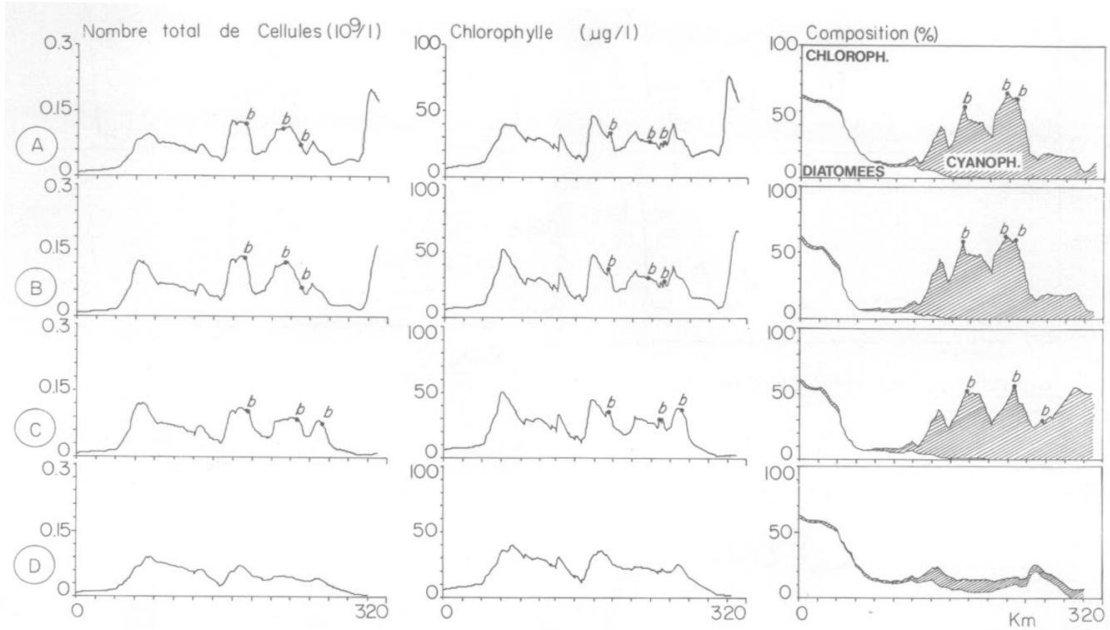

Fig. 5 : Variation de la composition phytoplanctonique en fonction de la période de modélisation (débit $10 \mathrm{~m}^{3 / \mathrm{s}}$ ). Dates de départ, $\mathrm{A}$ : ler juillet, $\mathrm{B}:$ ler aoùt, $\mathrm{C}:$ ler septembre, $\mathrm{D}:$ ler octobre. $(\mathrm{b}=$ conditions de bloom).

La figure 4 donne l'évolution des variables d'état les plus intéressantes dans le cas d'un débit régularisé de $10 \mathrm{~m}^{3 / \mathrm{s}}$. I] faut noter que les fluctuations des variables simulées présentent de fortes analogies avec les phénomènes observés, soit :

- le déphasage entre les concentrations de nutriments et les valeurs maximales de biomasse, avec un épuisement temporaire de la silice ou des nitrates (Dauta 1975), le maintien de faibles teneurs en PO4-P et l'apparition de $\mathrm{NH} 4 \mathrm{~N}$ lorsque le bloom disparait.

- le profil instable de chlorophylle, avec la production de fortes biomasses suivies de phases de déclin.

- une composition du phytoplancton où le développement des cyanophycées prend de l'importance à miparcours, avec une prédominance Chloro phycées-Cyanophycées vers l'aval.

- l'épuisement des nitrates favorise un bloom de cyanophycées au niveau des plus grands barrages Ruzech, Villeneuve, Le Temple). La mortalité qui accompagne la disparition du bloom libère les triments qui permettent aux autres espèces de reprendre la croissance.

Le développement propre à chaque espèce représentée dans le modèle peut être suivi : si certaines périodes correspondent à des phases communes d'essor ou de mortalité, le plus souvent chaque espèce montre un comportement typique de croissance.

Pour un débit de $10 \mathrm{~m}^{3} / \mathrm{s}$, le temps de transfert de l'eau à travers le systeme atteint presque 3 mois (Tableau I). La figure 4 donne une simulation pour une masse d'eau partant du $\mathrm{km}$ zéro au ler juin, et la figure 5 indique les résultats obtenus pour des parcours débutant à un mois d'intervalle (du ler juillet au ler octobre).

Pour ces simulations à débit constant $\left(10 \mathrm{~m}^{3 / 3}\right)$ correspondant à l'été et à l'automne, la même évolution amont-aval est retrouvée, avec des situations de bloom variables dans l'espace, et qui finissent par disparaitre en automne ( $f$ ig. $5 \mathrm{~d}$ ) où le déclin de la biomasse est dû aux conditions héliothermiques. 

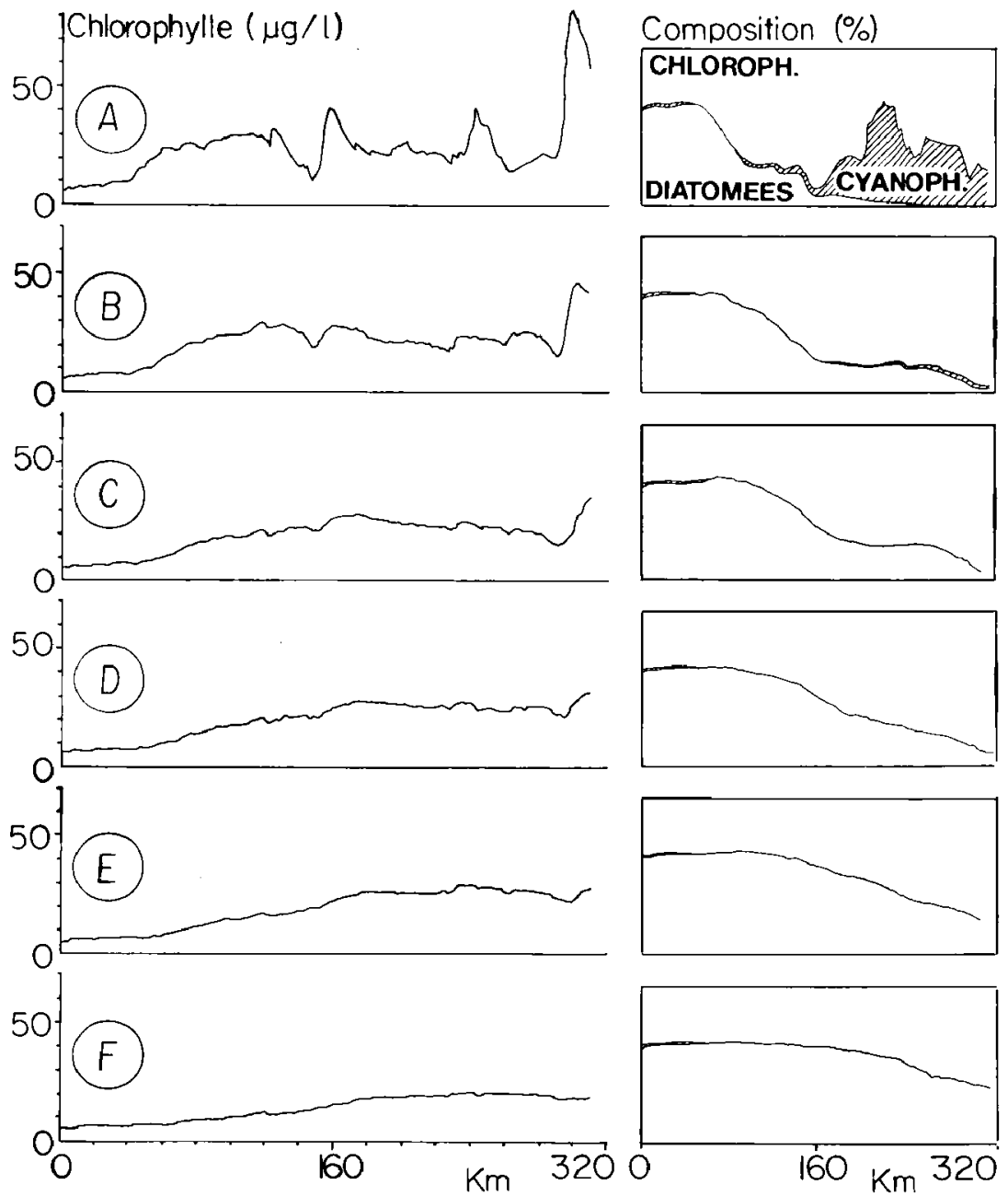

Fig. 6 : Evolution de la composition de la biomasse exprimée en chlorophylle, en fonction du débit. A : $10, B: 20, C: 30, D: 40, E: 50, F: 100 \mathrm{~m}^{3 / s}$. 

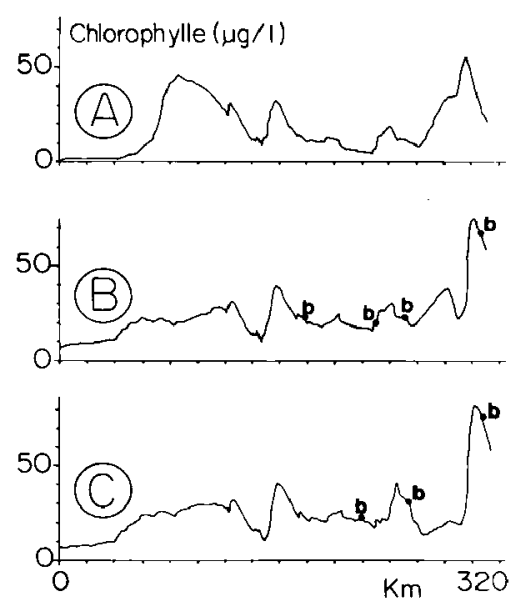

Fig. 7 : Comparaison de simulation réalisées avec 1(A), 3(B) et $8(\mathrm{C})$ especes d'algues. $(\mathrm{b}=$ conditions de bloom).

Tableau VII : Espèces d'algues composant la population phytoplanctonique modélisée, et composition initiale au km zéro: nombre de cellules par $\mathrm{ml}$.

\begin{tabular}{|c|c|c|}
\hline $\mathbf{N}^{\circ}$ espèce & Nom & Nombre de c \\
\hline 1 & Scenedesmus crassus & 2000 \\
\hline 2 & Coclastrum microsporum & 10 \\
\hline 3 & Dictyosphaerium pulchellum & 20 \\
\hline 4 & Scenedesmus quadricauda & 800 \\
\hline 5 & Pediastrum boryanum & 50 \\
\hline 6 & Monoraphidium minutum & 300 \\
\hline 7 & Anabaena cylindrica & 100 \\
\hline 8 & Fragillaria bidens & 5000 \\
\hline
\end{tabular}

L'augmentation du débit (fig. 6) a pour effet de modifier la composition de la population phyto planctonique avec:

- une réduction notable du développement des cyanophycées dont le rôle devient très faible pour un débit supérieur à $20 \mathrm{~m}^{3 /} \mathrm{s}$.

- un déplacement vers l'aval du maximum de biomasse algale et de la croissance des chlorophycées.
D'autre part, les conditions de bloom qui sont plusieurs fois réunies pour un débit de $10 \mathrm{~m} / \mathrm{s}$ n'apparaissent plus pour les débits supérieurs.

La figure 7 compare des simulations réalisées respectivement avec 1, 3 et 8 espèces d'algues, et pour un même débit, soit :

- 1 chlorophycée (esp. 1), fig. 7a

- 1 chlorophycée (esp. 2), diatomée (esp. 8), 1 cyanophycée (esp. 7), fig. 7b

- le cas normal (fig. 7c) prenant en compte les 8 espèces.

L'allure générale des profils longitudinaux est relativement semblable, et le cas comprenant 3 espèces est très proche du modèle le plus complexe. La simplification du nombre d'especes apporte une réponse d'autant plus marquée (croissance ou mortalité) que le nombre d'especes est réduit, avec à la limite (fig. 7a) une réponse typiquement liée aux caractères de l'espèce utilisée. Par contre, l'utilisation d'un modèle plurispécifique semble apporter une plus grande souplesse dans l'évolution de la population, et donne sans doute une meilleure approximation de la variation totale de biomasse : il est rare que les conditions soient simultanément défavorables pour toutes les espèces, de sorte que ce modèle est capable de générer des successions d'espèces proches de celles observées sur le terrain, chacune d'elles montrant un cycle de développement particulier en réponse aux facteurs environnementaux instantanés et antérieurs.

\section{5. - Discussion}

Elaboré à partir de résultats expérimentaux déterminés in vitro ou in situ, ce modele produit des simulations de dynamiques du phytoplancton qui présentent une forte analogie avec l'évolution longitudinale observée. En période estivale, les variations continuelles de débit (entre 5 et $50 \mathrm{~m}^{3 /} / \mathrm{s}$ ) créent un grand nombre de situations dont témoignent les profils longitudinaux enregistrées sur la rivière. Bien que les situations traitées par ce modèle soient purement fictives, et à cause de ceci une calibration était sans objet, la comparaison des résultats simulés et des phénomènes observés amène les remarques suivantes :

- l'évolution longitudinale du phytoplancton est marquée par une succession de phases de multiplication et de déclin, en relation plus ou moins directe avec les apports en éléments nutritifs. 
- la prédiction de l'épuisement des nutriments (NO3-N, PO4P, SIO2) concorde avec nos observations sur le terrain.

- la possibilité d'épuisement d'un ou plusieurs éléments nutritifs justifie le schéma d'un modèle comprenant trois principaux nutriments. Forsberg (1977) signale en effet que de plus en plus, dans les eaux eutrophes ou il y a rejet d'effluents, NO3-N semble devenir le facteur limitant le plus important.

- la prise en compte des réserves intracellulaires permet de prévoir la disparition quasi immédiate d'un apport dans le milieu, et d'autre part la prolifération différée du phytoplancton alors que le substrat est épuisé.

- l'utilisation d'un sous-modèle de type catastrophe déterminant les conditions de bloom de Cyanophycées semble qualitativement satisfaisante. Cependant, la calibration et la validation de ce type de formulation restent à faire plus précisément.

Ce type de modele permet donc de traiter la coexistence de plusieurs compétiteurs pour une même ressource, specialement lorsque la croissance est de type logistique, et en donnant une connaissance analytique de la structure des communautés. La résolution d'un modèle basé sur tune population plurispécifique confère au système une plus grande malléabilité, vraisemblablement plus proche de la réalité écologique, en offrant un plus grand nombre de " degrés de liberté " au développement d'une biomasse. Dans le modèle présenté, les Chlorophycées sont représentées par 6 espèces, contre 1 espèce pour les Diatomées et les Cyanophycées. Compte tenu de l'optimum thermique de la diatomée $\left(20^{\circ} \mathrm{C}\right)$, celle-ci est pénalisée lorsque la température s'élève (température moyenne estivale sur le cours inférieur comprise entre 24 et $29^{\circ} \mathrm{C}$, l'espèce disDarâit progressivernent vers l'aval de la rivièse sans étre remplacée, et la composition de la population diffère alors nettement des observations : une solution évidente consisterait à ajouter dans le modèle une diatomée comme Fragillaria crotonensis qui se développe en été.

Une des simplifications du modèle réside dans le fait que l'on considère la masse d'eau comme verticalement homogène. Or, pour les faibles débits d'étiage, il existe un gradient thermique vertical de plusieurs degrés correspondant à une stratification de l'oxygène dissous (Caussade \& al. 1978, Delclaux 1980). La prise en compte de ce phénomène modifierait notablement le rendement photosynthétique de la colonne d'eau. Nous avons pu montrer (Capblancq \& al. 1982) que le développement du phytoplancton est souvent limité par les conditions lumineuses : en raison de la turbidité, la production nette est nulle pour un quotient R/Popt compris entre 0,12 et 0,17 .

Cette demière remarque introduit le problèrne des paramètres encore mal connus, et remplacés par des sous-modèles approximatifs ou des constantes. Il s'agit principalement de la respiration, de la vitesse de sédimentation, de la biodégradation, des conditions d'apparition et de déclin de bloom de cyanophycées et des effets d'anabiose qui en résultent.

- A l'aide d'une analyse de sensibilité Simmons \& Lam (1980) notent à ce sujet que l'incertitude relative à la formulation de la sédimentation et de la régénération des nutriments amoindrit la capacité prédictive de leur modèle. La chute des cellules, couplée avec le taux de croissance (Scavia 1980) ou mise en relation avec l'état nutritionnel (Smayda 1970, 1974) ne peut être séparée des mouvements de circulation de l'eau. Titman et Kilham (1976) montrent que sur un lac stratifié, grâce aux cellules de Langnuir, il est possible d'expliquer le fait que les algues retrouvent un taux de croissance optimal tout en ayant une vitesse de chute élevée. Ce phénomène n'est pas mieux défini dans le cas d'un milieu lotique où la production de turbulences par le fond n'est pas toujours une garantie d'homogénéité du milieu à cause d'un gradient sol-fond (Caussade \& al. 1982).

- Les taux de reminéralisation de l'azote et du phos. phore, exprimés par des cinétiques du premier ordre reliées à la température (Scavia 1980) révèlent notre méconnaissance actuelle des effets réels du bactérioplancton : des tests de sensibilité (Capblancq \& al.1982) montrent que l'évolution simulée des concent rations en nutriments est grandement influencée par ces taux. Une remise à disposition rapide et permanente explique la possibilité d'avoir un phytoplancton proche d'un taux optimal de croissance malgré des concentrations non détectables en nutriments (Mc Carthy \& Goldman 1979).

Le modèle présenté permet d'aborder l'impact des apports à la rivière et de tester diverses hypothèses de gestion au niveau des rejets ou de l'hydraulique. Néanmoins, pour qu'un tel modèle soit réellement utilisable, une amélioration de la quantification des apports doit être développée. L'interconnection de sous-modèles d'érosion et de lessivage de bassin versant avec un modele biologique de ce type constitue l'une des prochaines étapes vers l'élaboration de modèles prévisionnels des impacts des activités humaines sur la qualité des caux de surface. 


\section{Travaux citès}

Angelier (E.), Bordes (J.-M.), Luchetta (J.C.) \& Rochard (M.) 1978. Analyse statistique des paramètres physicochimiques de la rivière Lot. Annls Limnol., 14 (1-2) : 39-57

Bierman (V.J.) 1976. Mathematical model of the selective enhan cement of blue-green algae by nutrient enrichment. In R.P. Canale (Ed.), Modelling Biochemical Processes in Aquatic Ecosystems. Ann Arbor Sciences Pub. : 1-31.

Booker (M.-J.) \& Walsby (A.-E.) 1981. Bloom formation and stratification by planktonic blue-green alga in an experimental water column. Br. Phyco. J., 16:411-421.

Capblancq (J.) Dauta (A.) 1978. Phytoplancton et production pri. maire de la rivière Lot. Annls Limnol, $14(1-2): 85-112$.

Capblancq (J.), Dauta (A.), Caussade (B.) \& Decamps (H.) 1982. Variations joumalières de la production du phytoplancton en rivière : modélisation d'un bief du Lot. Annls Limnol., 18(2) 101.132 .

Capblancq (H.) \& Decamps (H.) 1978. Dynamics of the phytoplank. ton in the river Lot. Verh. Intemat. Verein. Limnol., 20 1479.1484

Casanova-Batut (T.) 1977. Elude préliminaire en vue de la modélisation de l'écosystème Lot. Thèse 3ème Cycle, Toulouse, $109 \mathrm{p}$.

Caussade (B.) Chaussavoine (C), Dalmayrac (S.) \& Masbernat (L.) 1978. Modélisation d'écosvstèmes rivières : application à un bief du Lot. Annls Limmol. $14(1-2): 139-162$.

Chahuneau (F.), Des Clers (S.) \& Meyer (J.-A.) 1980. Les modètes de simulation en écologie lacustre. Présentation des différentes approches et analyse des modèles existants. Acta Oecolo gica., Oecol. Gener. 1(1): 27.50

Delclaux (F.) 1980. Production primaire en milieu thermiquement stratific. Modélisation et application à la rivière Lot. Thèse Doct. Ingénicur INP Toulouse, $177 \mathrm{p}$.

Dauta (A.) 1975. Etude du phytoplancton du Lot. Annis Limnol., 11 219.238

Dauta (A.) \& Capblancq (J.) 1985. Simulation numérique de la dyna. mique du phytoplancton dans une succession de réservoirs à faible temps de rétention sur la rivière Lot. Am/s Limmol. $21(3): 203-212$

Dauta (A.). Decamps (H.), Denat (D.), Galharague (J.), Giot (D.), Massio (J.-C.) \& Trollet (J.-L.) 1975. Etude de la turbidité et des matières en suspension dans les eaux du Lot et de leurs rela. tions avec les écoulement s. 3.- Evolution des matières en sus. pension et de la turbidité dans une masse d'eau marquée. Rapport BRGM, 75 S.G.N. 388 M.P.Y., 31 p.

Duckstein \&.), Casti (J.) \& Kenpf (J.) 1979. Modeling phytoplank. ton dynamics using catastrophe theory. Water Resour. Res., $15(5): 1189-1194$.

Fogg (G.-E.) 1971. Nitrogen fixation in lakes. Plant Soil (spec, vol.): $393-401$.
Forsberg (C.) 1977. Nitrogen as a growth factor in freswater. Prog. Water Technol. $8(4-5): 275-290$

Horne (A. J.). Sandusky (J.C.) \& Carmiggelt (C.J.W.) 1979. Nitro gen fixation in Clear Lake, California, 3. Repetitive synoptic sampling of the Spring Aphanizomenon blooms. Limnot. Oceanogr., 24 (2) : 316-318.

Klemer (A.-R.), Feuillade (J.) \& Feuillade (M.) 1982. Cyanobacterial blooms : carbon and nitrogen limitation have opposite effects on the buoyancy of Oscillatoria. Science, $215: 1629-1631$.

Kncechel (R.) \& Kalff (J.) 1975. Algal sedimentation : the cause of diatom blue-green succession. Verh. Internat. Verein. Limnol., 19 (1): $745-754$

Konopka (A.), Brock (T.-D.) \& Walsby (A.-E.) 1978. Buoyancy regulation by planktonic blue-green algae in Lake Mendota. Arch Hydrobiol., 83(4). 524-537

Kuentzel (L.-E.) 1969. Bacteria, carbon dioxide and algal blooms J. Water Pollut. Control Fed., $41:$ 1737-1747.

Lehman (J.-T.), Botkin (D.-B.) \& Likens (G.-E.) 1975. The assumptions and rationales of a computer model of phytoplankton population dynamics. Linmol. Oceanogr. 20(3) : 343-363.

Massio (J.-C.) 1976. Facteurs d'évolution des matières en suspension minérales et organiques dans les eaux du Lot. Thèse Jème cycle, Toulouse: $146 \mathrm{p}$

Mc Carthy (J . J.) \& Goldman (3.C.) 1979. Nitrogenous nutrition of marine phytoplankton in nutrient depleted waters. Science. $203(4381): 670-672$

Ogawa (R-E.) \& Carr (J_-F.) 1969. The influence of nitrogen on heterocyst production in blue-green algae. Limnol. Oceanogr., 14 342-351

Scavia (D.) 1980. An ecological model of lake Ontario. Ecol. Model. ling., 9(3): 233-245.

Simmons (J.-J.) \& Lam (D.-C.) 1980 . Some limitations of water quality models of large lakes : a case study of lake Ontario. Water Resout. Res., I6(1) : 105-116.

Smayda (T.J.) 1970. The suspension and sinking of phytoplankton in the sea. Oceanogr. Mar. Biol. Annu. Rev., $8: 353-414$

Smayda (T.-J.) 1974. Some experiments on sinking characteristics of two freshwater diatoms. Limnol Oceanogr., $19: 628.365$

S.O.G.R.E.A.H. 1975. Etiages du Lot, étude sur modèle mathématique de l'exploitation des usines. Document $n^{\circ} 11792$

Thomson (G.D.) 1979. A model for nitrate-nitrogen transport and denit ification in the river Thames. Water Res., 13(9): 855-863.

Titman (D.) \& Kilham (P.) 1976. Sinking in freshwater phy toplankton : some ecological implications of cell nutrient status and physical mixing processes. Limnol. Oceanogr., $21(3): 409-417$

Tourenq (J.N.), Capblancq (J.) \& Casanova (H.) 1978. Bassin versant et hydrobiologie de la rivière Lot. Annls Limnol., 14(1-2) 9.24.

Walsby (A.-E.) \& Booker (M.-J.) 1980. Changes in buoyancy of planktonic blue-green alga in response to light intensity. Br. Phycol J., $15: 311.319$. 